\title{
Aportes para pensar la relación educación-trabajo: el caso de la Escuela Universitaria de Oficios
}

Contributions to think about the education-work relationship: the case of the University School of Trades

\author{
María Eugenia Vicente \\ https://orcid.org/0000-0003-0748-678X \\ vicentealbisu@gmail.com \\ Instituto de Investigaciones en Humanidades \\ y Ciencias Sociales | Consejo Nacional de \\ Investigaciones Científicas y Técnicas | \\ Universidad Nacional de La Plata | Argentina
}

\author{
Alicia Inés Villa \\ https://orcid.org/0000-0003-0548-9928 \\ alivilla@gmail.com \\ Instituto de Investigaciones en Humanidades \\ y Ciencias Sociales, Consejo Nacional de \\ Investigaciones Científicas y Técnicas | \\ Universidad Nacional de La Plata
}

\section{RESUMEN}

El artículo permite observar las relaciones entre educación, trabajo e inclusión social a partir de un dispositivo específico: la Escuela de Oficios de la Universidad Nacional de La Plata. A partir de fuentes secundarias se analiza la propuesta educativa de la escuela en torno a cuatro ejes: I) su aporte a las políticas públicas de inclusión social, II) las trayectorias socieducativas de los usuarios y el fortalecimiento de carácter ciudadano, III) la estrategia de gestión y IV) Los lineamientos curriculares y de formación favorecedores de la inserción en el trabajo con inclusión. De manera más general, nos proponemos visibilizar el papel estratégico que juega la Universidad como un actor activo de la inclusión social, la educación de calidad y el trabajo genuino.

\section{ABSTRACT}

The article allows observing the relationships between education, work and social inclusion from a specific device: the School of Trades of the National University of La Plata. Based on secondary sources, the educational proposal of the school is analyzed around four axes: I) its contribution to public policies of social inclusion, II) the socio-educational trajectories of the users and the strengthening of citizenship, III) the strategy management and IV) The curricular and training guidelines favoring the insertion in the work with inclusion. More generally, we propose to make visible the strategic role that the University plays as an active actor of social inclusion, quality education and genuine work.

PALABRAS CLAVE educación y trabajo, inclusión social, universidad, Escuela de Oficios.

\section{KEY WORDS}

education and work, social inclusion, university, School of Trades. 


\section{INTRODUCCIÓN}

La relación educación y trabajo no remite únicamente a una relación de tipo económica, sino, principalmente social, evocando procesos y estrategias de (re)configuración del tejido social; anudando subjetividades, identidades, trayectorias de los sujetos, valores, creencias sostenidas y compartidas, en marcos estructurales e históricos (Castel,1997) Ambas relaciones, al plasmarse en instituciones educativas, plantea desafíos formativos, curriculares, proyectuales que deben considerar a sus usuarios, sus necesidades y su territorio.

\section{Proponemos aquí un recorrido descriptivo por las estrategias de vinculación entre educación y trabajo a partir de una polí- tica universitaria concreta, como lo es la Escuela Universitaria de Oficios (EUO) de la Universidad Nacional de La Plata (UNLP).}

Proponemos aquí un recorrido descriptivo por las estrategias de vinculación entre educación y trabajo a partir de una política universitaria concreta, como lo es la Escuela Universitaria de Oficios (EUO) de la Universidad Nacional de La Plata (UNLP). Al respecto, se destacan variados aportes al tema (Bonicatto, 2012; Bonicatto, Bermudez, Calvo y otros, 2012; Bonicatto, Serrichio, Arcidiacomo, Gallardo y Tiramontini, 2012; Gallardo, Moralejo y Vailati, 2013; Serrichio, 2015) En esta oportunidad, sin agotar las dimensiones que constituyen la trama socio educativa de la propuesta de la EUO, se ha realizado un recorte so- 
bre cuatro ejes que nos invitan a pensar las características principales de la Escuela. A saber:

I) El aporte de la EUO a las políticas públicas de inclusión social a través del fortalecimiento de la relación educación- trabajo que, desde la universidad, se orientan a sostener y resignificar el tejido social.

II) Las trayectorias socio educativas que se anidan, se despliegan y se sostienen en el seno de la propuesta de la EUO orientadas al vínculo entre educación y trabajo pensado en términos de participación territorial y democracia educativa.

III) Su estrategia de gestión. Ésta se encuadra en procesos sociales de coordinación y decisión, donde la horizontalidad y la recursividad reflexiva, son componentes característicos en el desarrollo de la propuesta.

IV) Los lineamientos de formación y curricular que, desde una posición integral y social del vínculo entre educación y trabajo, se construyen en propuestas de formación orientadas a una concepción del trabajo, la inclusión, la producción y los trabajadores amplia, en marcos de inclusión social.

\section{LA EUO EN EL MARCO DE UNA POLÍTICA PÚBLICA}

Es importante destacar el lugar particular que la UNLP otorga a las políticas integrales que pone a disposición del territorio donde la universidad se emplaza. Los recursos, saberes, tecnologías que se producen en la misma se ponen a disposición del territorio para su mejora y desarrollo. Las áreas de Extensión universitaria, la Pro Secretaría de Políticas Sociales y el Consejo Social constituyen dichas áreas, las que convocan a la participación de diversos actores, organizaciones e instituciones de la universidad.

Dentro de la Pro secretaría de Políticas Sociales se ubica la Escuela de Oficios como parte de esas políticas integrales, orientadas a potenciar la capacidad transformadora de las políticas públicas inclusivas, en la consolidación del pleno ejercicio de los derechos humanos (Amaya, 2018). El enfoque de derechos empodera a los sujetos ubicándolos como titulares de derechos que demandan prestaciones y conductas, y las políticas públicas se constituyen en la vía elegida por el Estado en su conjunto, orientado a la restitución de un equilibrio en las desigualdades (Abramovich, 2004). Si bien la universidad no reemplaza a las responsabilidades del gobierno en la implementación de políticas públicas, no obstante, se replantea de qué manera puede acompañar el abordaje de problemas de agenda pública, desde un aporte calificado y en vínculo con los equipos de gestión estatal y las organizaciones sociales (Bonicatto, 2012; López, 2009).

En este encuadre se concibe la dinámica de la EUO que pone en 
juego normas y recursos que, a su vez, tensionan valores y posicionamientos (Neirotti, 2017). En función de ello, la UNLP se posiciona en una clara consideración respecto de la necesidad de políticas sociales integrales, comprometidas con el ámbito local y reconstructoras del tejido social (Serrichio, 2015; Bonicatto, Bermudez, Calvo y otros, 2012; Estatuto, 2008). En este marco, en 2008, se crea la Prosecretaría de Políticas Sociales, cuya estructura organizacional se define a través de tres líneas estratégicas: gestión territorial, participación en el diseño e implementación de políticas públicas y fortalecimiento de la red de inclusión social. En esta última línea se encuadra la Escuela Universitaria de Oficios, que constituye un espacio educativo para la inclusión laboral, a través de la capacitación en oficios artesanales e industriales destinados a todas las edades y atendiendo a las situaciones particulares de escolarización y de trabajo previos (Serrichio, 2015).

El vínculo entre educación y trabajo que se vislumbra en la política de la EUO presenta características socio territoriales orientadas a formar una ciudadanía consciente de su contexto y con capacidad de intervenir significativamente en los procesos productivos. Desde una mirada superadora de la lógica de ajuste, se hace hincapié en las posibilidades que la propia formación ofrece: la emancipación. De esta manera, los ciudadanos en formación podrán no sólo insertarse al mundo laboral, sino hacerlo de forma consciente de sus intereses e historias, en el contexto territorial.

\section{El vínculo entre educación y trabajo que se vislumbra en la política de la EUO presenta características socio territoriales orientadas a formar una ciudadanía consciente de su contexto y con capacidad de intervenir significativamente en los proce- sos productivos.}

\section{TRAYECTORIAS SOCIO EDUCATIVAS}

Las trayectorias por el sistema educativo y el mundo del trabajo no son lineales, sino que se constituyen en el seno de diversos recorridos heterogéneos y variables aún en un mismo recorrido de vida, conformando procesos dinámicos en un tiempo determinado (Bidart y Brochier, 2010; Miranda, 2016). En este marco, se reconoce una mirada superadora del supuesto clásico que concibe a los mercados laborales como continuos, que la relación laboral es única y principalmente mercantil, y que el trabajador debe ajustarse al contexto laboral donde la obtención del título académico representa la interiorización en el individuo de valores y actitudes útiles al sistema 
productivo (Doeringer y Piore, 1971).

En este marco, la EUO se orienta a empoderar a los sujetos, partiendo de sus intereses, necesidades, problemas y demandas en orden a crear instancias y estrategias de transformación para sus inserciones laborales, involucrando la posibilidad de retomar estudios inconclusos. En función de ello, se plantea un esquema de formación gradual que se inicia en el barrio donde vive el joven o adulto, reconociendo la importancia de la pertenencia territorial para fortalecer confianzas y vínculos. En este sentido, el proceso de formación parte de un análisis de la situación de cada barrio y de cada grupo de jóvenes para ajustar la propuesta pedagógica. El equipo docente, que desarrolla el proceso de enseñanza- aprendizaje orientado a sostener las trayectorias socio educativas, se fortalece con un dispositivo de tutorías enmarcadas en el Centro de Orientación Vocacional Ocupacional, y el Programa de Vinculación y Orientación Laboral de la misma universidad. Las tutorías tienen como misión principal la de mantener la permanencia en las actividades propuestas por la EUO como, así también, promover que el estudiante pueda generar redes en su comunidad que lo sostengan aun después de finalizado el curso, y fortalecer la inclusión laboral de los estudiantes de la EUO (Gallardo, Moralejo y Vailati, 2013).

Luego del recorrido por la dimensión de trayectorias educativas y laborales, y las acciones de la EUO orientadas fortalecimiento de dichas trayectorias, es posible pensar la relación educación y trabajo a través de un concepto de trayectoria que remite a un proceso, que no sólo contiene pasos o etapas vitales, sino que refleja relaciones, intereses y estrategias de participación en el territorio social y productivo. En este marco, los capitales y el territorio juegan un papel fundamental y diferencial en las trayectorias de los trabajadores y estudiantes configurando diferentes recorridos a lo largo de sus vidas y condicionando o posibilitando sus vínculos con el sistema educativo y el mundo del trabajo. Es por ello que podemos pensar en una política sostenida por la EUO que sostiene una concepción de trayectorias sociales colectivas que, a partir del reconocimiento de las desigualdades educativas y el condicionamiento de la participación en el mercado laboral, se desarrolla una estrategia de formación que, partiendo de la significación del territorio, se orienta a sostener a los estudiantes en espacios colectivos en los que se tejen redes y vínculos con la comunidad desde el inicio y en instancias posteriores al cursado.

\section{GESTIÓN SOCIAL}

La gestión, en tanto componente primordial de la política de la EUO, adquiere una forma estratégica para el sostenimiento de una política pública de inclusión, desarrollada a través de las características propias de una gestión social. Se trata de un tipo de estrategia de gestión que, 
desde el ámbito educativo, busca desarrollar metodologías de trabajo colectivas adecuadas al contexto social y cultural de los territorios en los que se insertan las instituciones educativas (CFE, 2007).

\section{La gestión, en tanto componente primordial de la política de la EUO, adquiere una forma estratégica para el sostenimiento de una política pública de inclusión, desarrollada a través de las características propias de una gestión social.}

La implementación de la EUO fue definida como un espacio para ser co- gestionado entre los ámbitos académicos, organizaciones sociales y gubernamentales interesados en la construcción de alternativas a las problemáticas de inserción educativas y laborales. Se plantea así un trabajo en conjunto desde áreas de investigación y formación pertenecientes a la UNLP y las organizaciones que podrían involucrarse, como ONGs y sindicatos, la Dirección General de Escuelas a través de la Dirección de Formación Profesional, los diferentes Municipios de La Plata y alrededores, la Comisión de Investigaciones Científicas de la Provincia de Buenos Aires y la Escuela Naval Universitaria. Particularmente, se ha logrado constituir convenios de cooperación con Clubes de la Ciudad de La Plata, industrias y asociaciones de diferente orden. Así, la cooperación entre organizaciones ha permitido complementar esfuerzos y dar cuenta de las preocupaciones sobre la temática desde perspectivas y lógicas diferentes (Bonicatto, Bermudez, Calvo y otros, 2012).

Luego del recorrido por la dimensión de gestión que configura la propuesta de la EUO, se reconoce una estructura basada en la horizontalidad en las relaciones entre los actores que forman parte de estos espacios educativos y la organización comunitaria como características distintivas de las estrategias de gestión social. La estrategia de co-gestión también permite visualizar la posibilidad de la reconstrucción del tejido social, vinculando el ámbito social, laboral y educativo en el seno de una intervención organizacional que involucra a los diferentes actores haciéndolos partícipes de la propuesta de formación para el trabajo.

\section{LA FORMACIÓN Y EL CURRICULUM}

Las características de la formación y proyecto curricular se constituyen en dimensiones relevantes y necesarias al momento de trazar las características socio educativas de la propuesta de la EUO. En este sentido, la preocupación principal de la Escuela es identificar aquellas situaciones que repercuten negativamente en el sostenimiento de la participación de los inscriptos a los cursos, interpelando el desarrollo 
de prácticas de enseñanza dirigidas a jóvenes y adultos que transitan los trayectos formativos de la EUO (Bonicatto, Serrichio, Arcidiacomo, Gallardo y Tiramontini, 2012). Desde este encuadre, el curriculum es entendido como un diseño en el que se juegan un conjunto de elementos culturales, valores, creencias, hábitos que conforman una política educativa (De Alba, 1998). En función de ello, el proceso de formación se define a través de diseños curriculares que superan la concepción tradicional donde los contenidos de enseñanza y se limitan a una simple reducción descendente de un saber universitario. Más bien, en la formación como práctica constitutiva de sujetos, tanto educadores como educandos se repiensan mutuamente, en términos de quién ocupa el espacio del conocimiento, quién detenta la concentración del saber y la capacidad de transmitirlo, quién tiene el derecho a legitimar o deslegitimar los códigos que se transmiten en los cursos (Villa, 2020). En este sentido, el curriculum se configura a través de prácticas sociales de referencia (Astolfi, 2001) que legitiman y hacen lugar a una gran variedad de prácticas sociales que presentan de distintas maneras una misma idea. Desde ese punto de vista, el saber erudito sólo es una práctica de referencia y también son considerados los saberes provenientes de prácticas culturales, laborales, socio comunitarias, para llegar a una formulación diversa de los conceptos a enseñar.

En este marco, la EUO, en su dimensión formativa y curricular, se orienta al fortalecimiento de derechos desde la capacitación a través de diseños educativos situados (Gallardo, Moralejo y Vailati, 2013). Para ello, la formación se piensa en orden a garantizar el ejercicio del derecho a una educación democrática, que incluya la participación de la comunidad en el proceso de enseñanza-aprendizaje. Como producto de ello, la Escuela propone un conjunto de estrategias que sostienen la propuesta de formación: a) La dupla pedagógica docente/tutor/a, que acompaña al estudiante en la permanencia en las actividades propuestas y aporta a la elaboración de futuros proyectos ocupaciones y formativos. b) La caja de herramientas, un conjunto de elementos imprescindibles para desarrollar el oficio, fortaleciendo su autonomía y viabilizando su actividad laboral. c) La entrega de recursos gratuitos, como material didáctico, de lectura, útiles, refrigerio, carga de tarjeta de transporte. d) El diseño de trayectos formativos que, como se mencionó en apartados anteriores, inician su recorrido en los barrios y culminan en las aulas de la UNLP, que se extiende hasta mantener lazos de apoyo a la inserción laboral luego de finalizado el curso.

\section{Para ello, la formación se piensa en orden a garantizar el ejer- cicio del derecho a una educación democrática, que incluya la participación de la comunidad en el proceso de enseñan- za-aprendizaje.}


Este conjunto de estrategias sostiene un trayecto de formación modular que comienza en un primer nivel introductorio de "Formación de capacidades básicas", dedicado a la recuperación y nivelación de saberes previos, a la consolidación del vínculo pedagógico y al entramado relacional entre los estudiantes del curso. Se continúa con un segundo nivel de cursos de "Formación profesional de nivel inicial", en el que los graduados del primer nivel continúan su formación en los Centros de Formación Profesional más adecuados a su perfil. En este nivel se alcanza la matriculación para aquellas disciplinas que así lo requieran. Finalmente, un tercer nivel de cursos la constituye la "Formación profesional continua", que permite el avance de contenidos en niveles de complejidad creciente en el marco de lo previsto por el Consejo Federal de Educación para la formación de oficios. Finalizados los módulos de formación, la UNLP reconoce a través de un certificado de competencias avalado por el Ministerio de Educación de la Nación, la capacitación recibida en la orientación seleccionada.

En este sentido, la dimensión de formación y curricular de la EUO, basada en procesos de co-gestión y acuerdos, remiten a saberes a enseñar que se construyen activa y colectivamente, constituyendo un proceso de axiologización que alcanza a la reflexión comunitaria sobre las finalidades y valores que pretenden trasmitirse a través de la enseñanza. Asimismo, se trata de un proceso de didactización vinculado a una construcción co gestiva del saber que permite "crear lo enseñable" (Astolfi, 2001). Con ello se demuestra que, en términos curriculares, no basta una definición para construir un saber, sino también se necesita del nucleamiento de diferentes miradas, posiciones y voces para estudiar cómo puede funcionar dicho saber y de disponer de los instrumentos materiales e intelectuales correspondientes de tal manera que el saber producido en el curso y al final de la actividad, permita responder al problema planteado.

\section{REFLEXIONES FINALES}

El recorrido por estas páginas pretendió mostrar las estrategias de vinculación entre educación y trabajo a partir de una política universitaria concreta, como lo es la Escuela Universitaria de Oficios. Recorrer la complejidad de la propuesta en estas breves páginas ha sido un desafío y, consideramos, no haber agotado las conceptualizaciones sobre la propuesta de la Escuela.

De todos modos, hemos intentado recortar la descripción en un conjunto de lineamientos que, a nuestro parecer, son centrales para vislumbrar la potencia e integralidad de esta política de inclusión social. En función de ello, el artículo invita a recorrer las tramas entre educación y trabajo que se entretejen desde la extensión social de la Universidad Nacional de La Plata, a partir de una definición de política 
pública orientada a la reconstrucción del tejido social en escenarios productivos comunitarios. Esta orientación se acompaña de una estrategia colectiva de gestión donde tienen lugar los diferentes actores educativos, productivos y sociales, sosteniendo una propuesta de formación consciente de la construcción de un saber a enseñar anclado territorialmente y orientado a la democratización de las trayectorias educativas y laborales de los sujetos.

En función de ello, el artículo invita a recorrer las tramas entre educación y trabajo que se entretejen desde la extensión social de la Universidad Nacional de La Plata, a partir de una definición de política pública orientada a la reconstrucción del tejido social en escenarios productivos comunitarios.

Esta propuesta no está exenta de variables que la interpelan y desafían, como el sostenimiento de políticas de inclusión de los diferentes gobiernos nacionales, las posibilidades presupuestarias dedicadas a la extensión universitaria, los índices de retención de los estudiantes en los cursos, la virtualización de la propuesta en contexto pandémico, entre otros. De todos modos, el aporte central es conocer aquellas intervenciones al sector educativo y laboral que generan posibilidades de pensar y desarrollar acciones que, desde la universidad, nos incluya a todos. 


\section{BIBLIOGRAFÍA}

Abramovich, V. (2004). Una Aproximación al Enfoque de Derechos en las Estrategias y Políticas de Desarrollo de América Latina. CELS (Centro de Estudios Legales y Sociales, Argentina). Derechos y Desarrollo en América Latina: Una Reunión de Trabajo. Santiago, Chile.

Amaya, P. (2018). Capacidades institucionales y evaluación de políticas: bases para construir al Estado. XIII Congreso Nacional y VI Congreso Internacional sobre Democracia. Facultad de Ciencia Política y Relaciones Internacionales. Universidad Nacional de Rosario.

Astolfi, J. P. (2001) Conceptos clave en la Didáctica de las Disciplinas. España: Díada.

Bidart, C. y Brochier, D. (2010) "Las bifurcaciones como cambios de orientación en un proceso" en Ariel Mendez (Comp.) Procesus, Concepts et méthode pour l'analyse temporelle en Sciences Sociales. Belgique: Academia Bruylant.

Bonicatto, M. (2012) (Comp.) Primeras Jornadas Nacionales de Políticas Públicas y Universidad. Secretaría de Extensión, UNLP, La Plata.

Bonicatto, M., Bermúdez, L., Calvo, M. V., Iparraguirre, M., Moratti, F., Serrichio, S. y Tiramontini, M. (2012) La Universidad en la implementación de Políticas Públicas. X Jornadas Nacionales de Extensión Universitaria y II Jornadas Regionales de Extensión Universitaria, Neuquén.

Bonicatto, M., Serrichio, S., Arcidiacomo, M., Gallardo, J. y Tiramontini, M. (2012). La Escuela de Oficios de la UNLP. Fortalecimiento de derechos desde la capacitación. 5to Congreso Nacional de Extensión Universitaria, Córdoba.

Castel, R. (1997) La metamorfosis de la cuestión social. Una crónica del salariado. Buenos Aires: Paidós.

CFE (2007) Escuelas de gestión social. Resolución CFE N³3/07, Consejo Federal de Educación.

De Alba, A. (1998) Curriculum: crisis, mito y perspectivas. Buenos Aires: Miño y Dávila.

Doeringer, P. B.; Piore, M. J. (1971) Internal Labor Markets and Manpover analysis. DC: Hert and Co.

Enguita, M. (2011) Del desapego al desenganche y de este al fracaso escolar. Cad. Pesqui. vol.41, num.144, pp. 732- 751. 
Estatuto de la Universidad Nacional de La Plata, 2008. Disponible en: http://www.fahce.unlp.edu.ar/normativa/estatuto-de-la-universidad-nacional-de-la-plata

Gallardo, J. Moralejo, D. y Vailati, Y. (2013) La orientación laboral en el marco de la Escuela Universitaria de Oficios de la Universidad Nacional de La Plata. $4^{\circ}$ Congreso Internacional de Investigación de la Facultad de Psicología de la UNLP. Conocimiento y práctica profesional: perspectivas y problemáticas actuales, La Plata.

López, N. (Coord). (2009). De relaciones, actores y territorios: hacia nuevas políticas en torno a la educación en América Latina. Buenos Aires: IIPE-Unesco.

Miranda, A. (2016) Transiciones juveniles, generaciones sociales y procesos de inclusión social en Argentina post-neoliberal. Linhas Críticas, vol. 22, núm. 47, pp. 130-149.

Neirotti, N. (2017). Prólogo. En María Cristina Ruiz del Ferrier. (Comp). El control de Políticas Públicas. La cuestión de la transparencia y la transparencia en cuestión. (13-17). FLACSO, Buenos Aires.

Serrichio, S. (2015) Entrevista al Director de la Escuela Universitaria de Oficios. Trayectorias Universitarias, vol.1, num. 1, pp. 69-75.

Villa, A. I. (2020) Educación Formal Alternativa: jóvenes, adultxs y territorios. Confluencia de saberes, num. 2, pp. 31- 52. 